\title{
RECUERDO Y ACTUALIZACIÓN DE LAS BASES ANATÓMICAS DEL PENE
}

\author{
Raúl Vozmediano Chicharro' y Ramón Bonilla Parrilla².
}

\author{
'Servicio de Urología. Hospital Carlos Haya. Málaga. \\ 2Servicio de Urología. Hospital Clínico Universitario Virgen de la Victoria. Málaga. España.
}

\begin{abstract}
Resumen.- El conocimiento de la anatomía del pene es fundamental para realizar un correcto diagnóstico y enfoque del tratamiento más adecuado, de las distintas enfermedades que puede presentar: estenosis de uretra, disfunción eréctil, incurvación, congénita o adquirida, etc.; siendo imprescindible su conocimiento anatómico para un buen manejo quirúrgico.
\end{abstract}

El pene es el órgano masculino involucrado en la función miccional y sexual. El cuerpo del pene esta compuesto por tres cuerpos eréctiles, léase las estructuras profundas: los cuerpos cavernosos y el cuerpo esponjoso, este último rodea y cubre la uretra. La fascia de Buck se relaciona con las estructuras profundas del pene. La fascia superficial formada por tejido más areolar, el dartos, se relaciona con la piel y la vascularización.

La irrigación de las estructuras profundas depende de la arteria peneana común, rama de la arteria pudenda

\section{CORRESPONDENCIA}

Ramón Bonilla Parrilla Servicio de Urología Hospital Clínico Universitario Virgen de la Victoria Campus Universitario de Teatinos $\mathrm{s} / \mathrm{n}$ 29009 Málaga (España).

ramon.bonilla@uaims.net interna. La sangre del pene drena a través de 3 sistemas venosos: el superficial, el intermedio y el profundo.

Los nervios peneanos son ramas de los pudendos y los cavernosos. Los nervios pudendos son los encargados de la inervación somática motora y sensitiva. Los nervios cavernosos son una combinación de fibras aferentes parasimpáticas y simpáticas, y corresponden a los nervios del sistema autónomo del pene.

Palabras clave: Anatomía pene. Disfunción eréctil.

Summary.- OBJECTIVES: The knowledge of penile anatomy is basic to perform a proper diagnosis and direct the most adequate treatment of the various diseases that may appear: urethral stenosis, erectile dysfunction, congenital or acquired penile curvature, etc.; being its anatomical knowledge essential for a proper surgical management.

The penis is the male organ involved in both voiding and sexual functions: the body of the penis is composed by three erectile bodies, (i.e the deep structures): the corpora cavernosa and the corpus spongiosum, this last surrounding and covering the urethra. Buck's fascia is in relation to the deep structures of the penis. The superficial fascia, dartos, is made up from a more areolar tissue and is in relation to skin and vessels. The vascularization of the deep structures comes from the common penile artery, a branch of the internal pudendal artery. Penile blood drains through three venous systems: superficial, intermediate and deep systems. Pudendal nerves are in charge of the sensitive and motor somatic innervations. Cavernosal nerves are a combination of parasympathetic and sympathetic afferent fibers, corresponding to the nerves of the autonomic system of the penis.

Keywords: Penile anatomy. Erectile dysfunction. 


\section{INTRODUCCIÓN}

El pene es el órgano masculino que permite la micción y las relaciones sexuales. Es un órgano cilíndrico que pende sobre las bolsas escrotales, por debajo de la sínfisis pubiana (1). Está unido a la región anterior del periné. Su tamaño y consistencia varían según se halle en estado de flaccidez o de erección: en estado fláccido mide unos $8-10 \mathrm{~cm}$ y en erección se vuelve rígido y mide unos $14-15 \mathrm{~cm}$.

El cuerpo del pene se encuentra formado por tres cilindros eréctiles, junto a sus planos fasciales, sus nervios y su vascularización, todo ello recubierto por piel. Los tres cilindros eréctiles que están revestidos por la fascia de Buck, son los cuerpos cavernosos y, en posición ventral, el cuerpo esponjoso que en su interior aloja la uretra. La fascia de Buck es una capa elástica y resistente inmediatamente adyacente a la túnica albugínea (2) (Figura 1).

Los cuerpos cavernosos constituyen la mayor parte del pene y se inician en las ramas isquiopubianas (3). Contienen el tejido eréctil en el interior de una densa vaina elástica de tejido conectivo, la túnica albugínea. Los cuerpos cavernosos no son estructuras independientes, si no que constituyen un único espacio a través de un tabique incompleto en la línea media, el septum o septointercavernoso. Este tabique se muestra más completo hacia la base del pene, convirtiéndose en un tabique completo cuando los cuerpos cavernosos se independizan y se dividen para formar los pilares, que están fijados a la rama inferior del pubis y el isquion. El tejido eréctil contiene arterias, nervios, fibras musculares y senos venosos revestido por células endoteliales planas, llenan el espacio de los cuerpos cavernosos y simulan al corte una esponja, cuyos huecos forman lagos sanguíneos. Los capilares sanguíneos rellenan estos huecos y están dotados de dispositivos musculares que permiten o cierran el paso de la sangre. Cuando la sangre pasa a estos lagos, los cuerpos cavernosos y esponjoso se hinchan y se endurecen. Las dos arterias cavernosas discurren cerca del centro de los cuerpos cavernosos. El flujo de sangre a través de estas arterias retorna a través

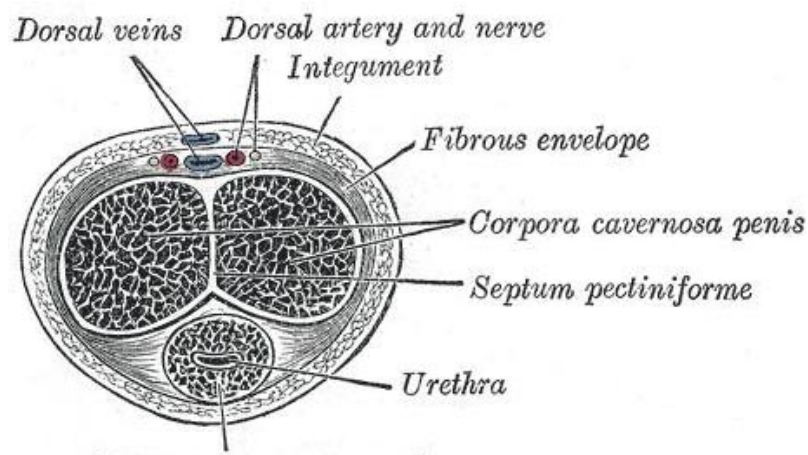

Corpus cavernosum urethree del espacio eréctil que se conecta a través de canales anastomóticos con el cuerpo esponjoso y con el drenaje venoso (4).

El cuerpo esponjoso (Figura 2) se sitúa en el surco ventral, entre los dos cuerpos cavernosos. El cuerpo esponjoso se fija bajo el músculo transverso profundo del periné, en un ensanchamiento denominado bulbo, recibiendo a partir de este punto a la uretra. El bulbo está recubierto por el músculo bulbocavernoso, con el denominado bulbo esponjoso en el centro. Los músculos isquiocavernosos y bulboesponjosos con sus conexiones con el elevador del ano parecen ejercer una acción importante en la erección. La uretra recorre todo el pene alojada dentro del cuerpo esponjoso. El extremo distal del cuerpo esponjoso se expande para formar el glande, un capuchón ancho de tejido con capacidad eréctil que cubre los extremos distales de los cuerpos cavernosos. Una de las funciones del cuerpo esponjoso es la de prevenir la compresión de la uretra durante la erección $(1,3)$.

En su base el pene esta sostenido por dos ligamentos, el ligamento suspensorio y el ligamento fundifor-

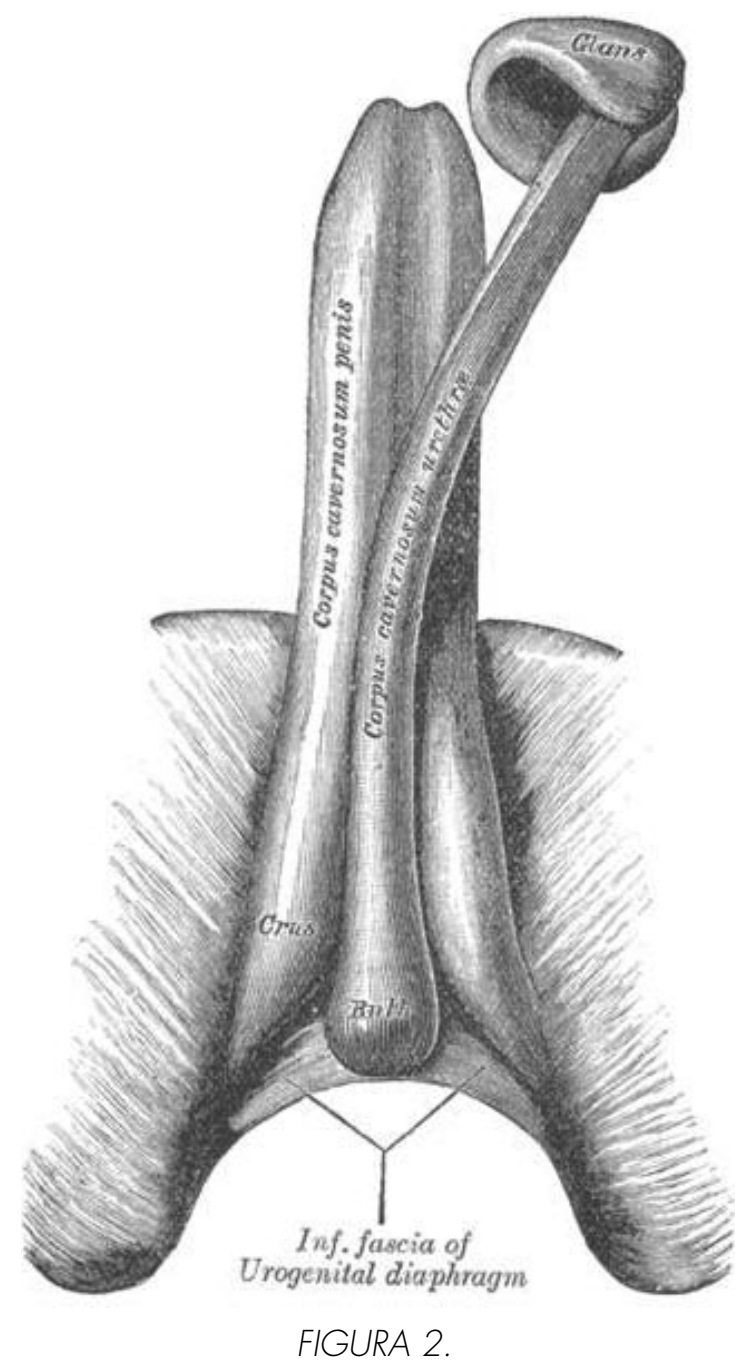




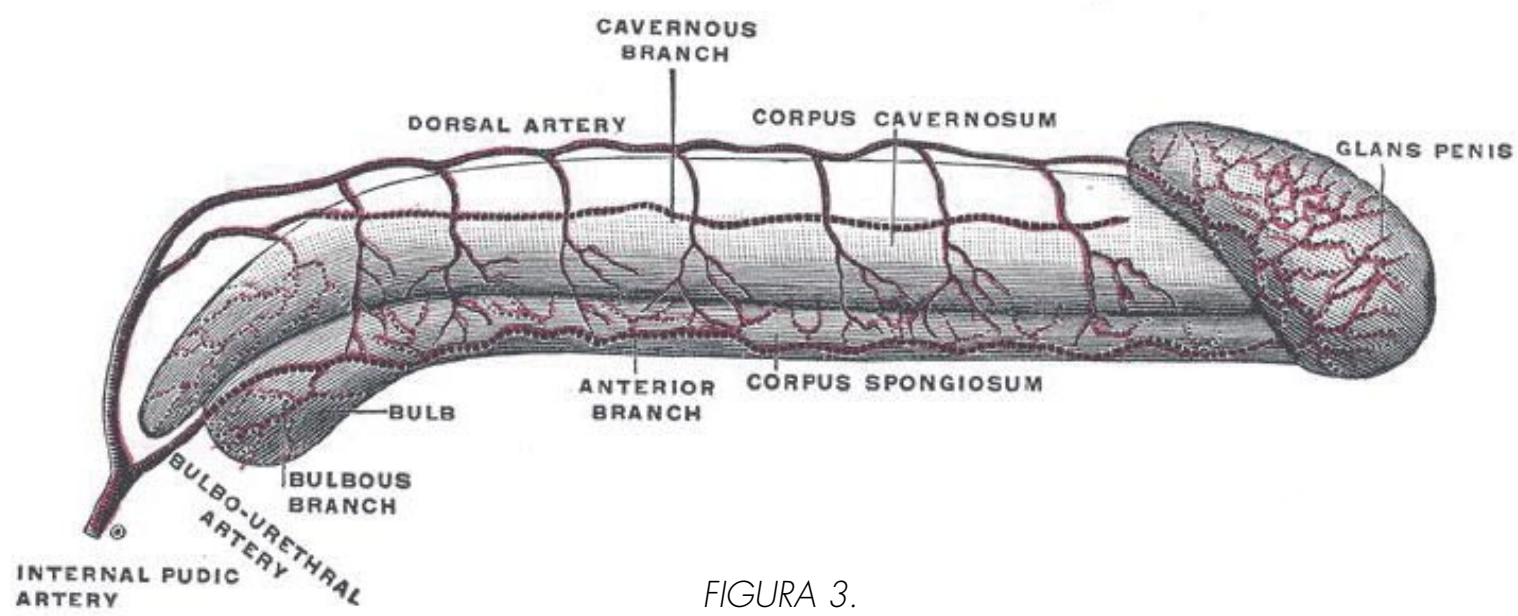

me superficial, que se continúan con la fascia del pene, y sujetan la zona medial de ambos cuerpos a dicha estructura ósea.

El pene está recubierto por varias capas; la más interna es una envoltura fibroelástica, la fascia peneana, que se continúa con la fascia superficial del escroto y periné. Esta envoltura se una a la sínfisis del pubis por el ligamento suspensorio del pene. El músculo dartos del escroto se continúa también por el pene formando otra de sus envolturas, entremezclándose con el tejido celular. La piel, con un tejido celular muy laxo, está adherido al pene en toda su longitud, excepto en el glande, con el cual sólo se une mediante una línea por su cara inferior denominada frenillo. El resto de la piel del glande está libre, cubriéndolo únicamente en estado de flaccidez. Esta porción de piel es el prepucio, que se retrae descubriendo el glande durante la erección $(1,3)$.

\section{VASCULARIZACIÓN DEL PENE}

\section{SISTEMA ARTERIAL (Figuras 3 y 4 )}

Generalmente, en la mayoría de los hombres la vascularización peneana proviene de la arteria pudenda interna, rama de la arteria iliaca interna. En un pequeño porcentaje de varones la vascularización puede provenir de las arteria obturatriz o isquiáticas (1).

En un porcentaje alto de varones 1 se puede observar una arteria pudenda accesoria originaria de la artería obturatriz, vesical, vesicoprostática o femoral.

La arteria pudenda interna recorre el conducto de Alcock saliendo de la pelvis por el agujero sacrociático mayor y penetrando por el agujero sacrociatico menor dirigiéndose hacia el canal pudendo. Una vez allí se denomina arteria peneana común y pasa a dividirse en sus ramas terminales (1):

- Arteria bulbouretral (también conocida como arteria del bulbo del pene, perineal profunda o transversa del perineo): es la primera rama terminal de la pudenda interna, y perforan la fascia de Buck para entrar en el musculo bulboesponjoso, se introduce en el bulbo, y dan lugar a ramas anteriores para los cuerpos cavernosos y posteriores para el bulbo (ramos bulbares y uretrales). Esas arterias están orientadas casi paralelas la uretra membranosa.

- Arteria dorsal del pene: dirigiéndose hacia el glande por el dorso del pene, se encuentra debajo de la fascia de Buck situado por fuera de la vena dorsal profunda del pene. Irriga el glande, la corona del glande y el prepucio del pene

- Arteria cavernosa o media del pene: penetra en los cuerpos cavernosos. Estas dan lugar a dos tipos de ramas:

o Helicinas o cortas (arteria de Mueller): se abren directamente en los espacios cavernosos sin formar capilares.

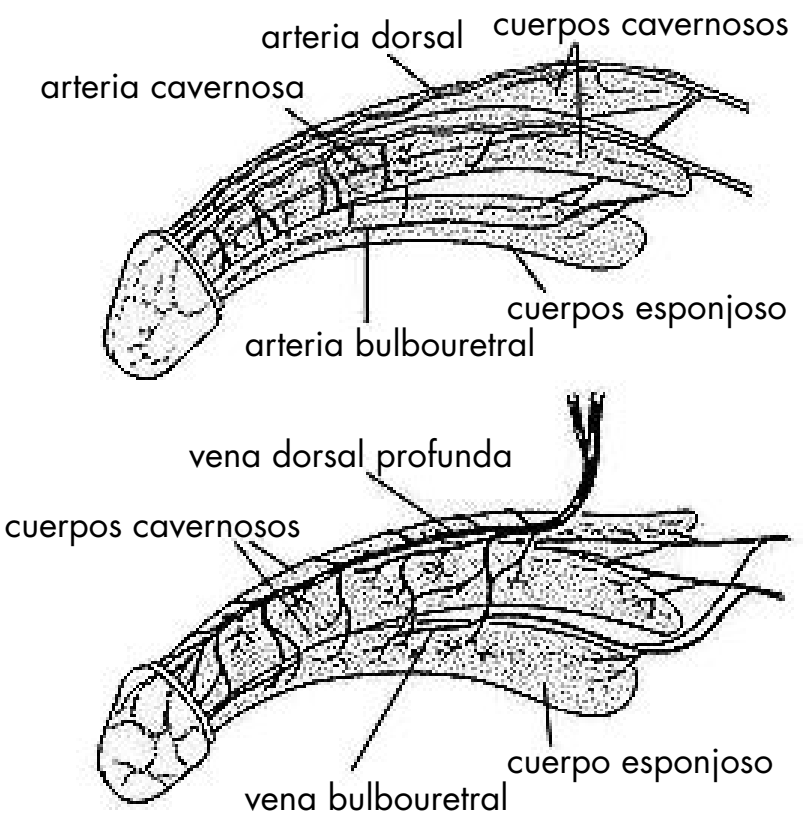

FIGURA 4. 
- Largas, que alcanzan el cuerpo esponjoso constituyendo shunt espongiocavernosos.

\section{DRENAJE VENOSO (Figura 4 y 5 )}

Se puede dividir en sistema superficial, intermedio y profundo (5).

- Sistema superficial: drenan la sangre de las envolturas del pene, prepucio y parte del glande. Dan lugar a la vena dorsal superficial del pene que drena en la pudenda externa, la cual es rama de la safena interna, habitualmente en la izquierda.

- El sistema intermedio se localiza por debajo de la fascia de Buck, pero por encima de la túnica albugínea. Recoge la sangre del glande, cuerpo esponjoso y de los dos tercios distales de los cuerpos cavernosos. Las venas del glande forman el plexo retrocoronal, que drena en la vena dorsal profunda del pene, así como las que provienen de los cuerpos cavernosos, las venas emisarias y las venas circunflejas, que atraviesan la túnica albugínea. Es frecuente las conexiones entre la venas dorsal superficial y la vena dorsal profunda. La vena dorsal profunda del pene transcurre por la línea media, desembocando en el plexo periprostático de Santorini.

- Sistema profundo: esta constituido por las venas de los pilares y cuerpos cavernosos, y nacen directamente de los espacios sinusoidales debajo de la albugínea formando las venas emisarias que atraviesan la misma

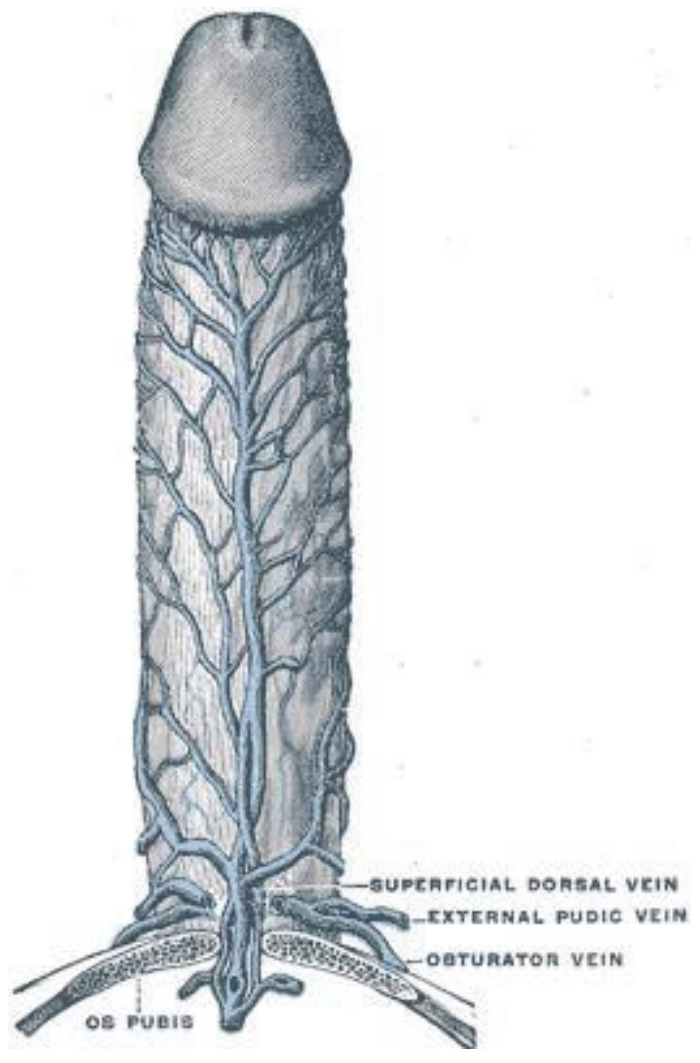

FIGURA 5. uniéndose al drenaje del cuerpo esponjosos formando las venas circunflejas y drenando en la vena dorsal profunda del pene que sigue un recorrido por la línea media entre los cuerpos cavernosos, drenando en el plexo de Santorini. El tercio proximal del pene (cuerpos cavernosos y cuerpo esponjoso) drena mediante las venas cavernosas y crurales hacia la vena pudenda interna y el plexo de Santorini.

\section{INERVACIÓN DEL PENE (Figura 6)}

\section{Sistema periférico}

La vascularización arterial y venosa, las fibras musculares intrínsecas del pene y la musculatura estriada regional están sometidas a un triple control del sistema nervioso periférico: el sistema simpático, el sistema parasimpático y el somático. Estas múltiples vías, tanto espinales como supraespinales, ejercen el control sobre este sistema periférico (4).

Los nervios que inervan al pene son ramas de los nervios pudendos y nervios cavernosos. Los nervios pudendo son los responsables de de la inervación motora y sensitiva somática al pene. Los nervios cavernosos son una combinación de las fibras aferentes parasimpáticas y simpáticas, y constituyen los nervios autonómicos del pene. Son éstos nervios los responsable de la inervación al componente eréctil del pene (4).

\section{Inervación somática}

La anatomía del nervio pudendo ha sido abundantemente descrita por la mayoría de los anatomistas, pero los datos electrofisiológicos nos muestran que los orígenes de las distintas ramas son bastante diferentes de lo que nos dice la anatomía convencional de acuerdo con las investigaciones anatomo-neurofisiológicas de Bischop y col (6).

El nervio pudendo está compuesto por fibras eferentes que inervan los músculos isquiocavernosos, bulbocavernoso y otros músculos estriados de la pelvis y el periné, y por fibras aferentes que proporcionan diversas estructuras del pene. Las neuronas motoras se encuentran en el núcleo de Onuf, en el segundo, tercero y cuarto segmento de la médula sacra. Los nervios pudendos entran en el periné con los vasos pudendos internos, a través de la escotadura ciática menos, en el borde posterior de la fosa isquiorrectal, entrando en el canal pudendo de Alcock, medial al musculo obturador interno. Tras proporcionar la rama que inerva el nervio rectal inferior se divide en el nervio perineal y el nervio dorsal del pene. El nervio perineal tiene ramas que inervan los músculos perineales, el esfínter uretral y parte del esfínter externo, y una rama que alcanza el cuerpo esponjoso. El nervio dorsal del pene perfora el músculo transverso perineal, entrando en el dorso del pene, donde se continúa distalmente a lo largo de la superficie dorsolateral del pene, lateral a la arteria dorsal. El nervio dorsal del pene constituye la vía aferente 
de la erección refleja, y lleva los impulsos desde la piel del pene, prepucio, frenillo, glande y del conectivo del septo intercavernoso hasta la medula (6-7).

El nervio pudendo es la vía sensitiva principal del pene, provocando su eliminación un bloqueo de la erección refleja evocada por la manipulación del pene.

Se ha demostrado en muchos modelos animales y en humanos que la estimulación eléctrica de los nervios cavernosos produce erección pero sin rigidez completa (7). La estimulación táctil del pene durante la actividad sexual pone en marcha el reflejo bulbocavernoso, que es un reflejo espinal en que los receptores se encuentran en el glande y los músculos involucrados son el bulbo y los isquiocavernosos. El reflejo bulbo cavernoso puede ser importante para alcanzar la máxima rigidez para y durante la penetración.

\section{Inervación simpática}

El centro simpático o toracolumbar, se encuentra en los segmentos medulares T-10 a L3. Las fibras preganglionares salen de la médula a través de los nervios espinales hacia la cadena simpática paravertebral, estableciendo sinapsis principalmente en el plexo hipogástrico superior, situado sobre los grandes vasos, a nivel de la $3^{\circ}$ vértebra lumbar y $1^{\underline{9}}$ sacra. En ocasiones las fibras preganglionares alcanzan este plexo hipogástrico a través de los nervios esplácnicos lumbares $(1,8)$.

El plexo hipogástrico superior se divide en dos nervios hipogástricos, que desembocan en el espacio pélvico. Estos nervios tienen fibras postganglionares procedentes del plexo hipogástrico y fibras preganglionares que harán sinapsis en el plexo pélvico. Las fibras postganglionares de los plexos hipogástricos pélvicos y de las cadenas ganglionares para-vertebrales forman el nervio cavernoso, que atraviesa el pene y que es identificable cerca de la próstata. Recientes trabajos han puesto de manifiesto que la estimulación del tronco simpático, nervios hipogástricos o de las fibras simpáticas del nervio pudendo producen detumescencia peneana.

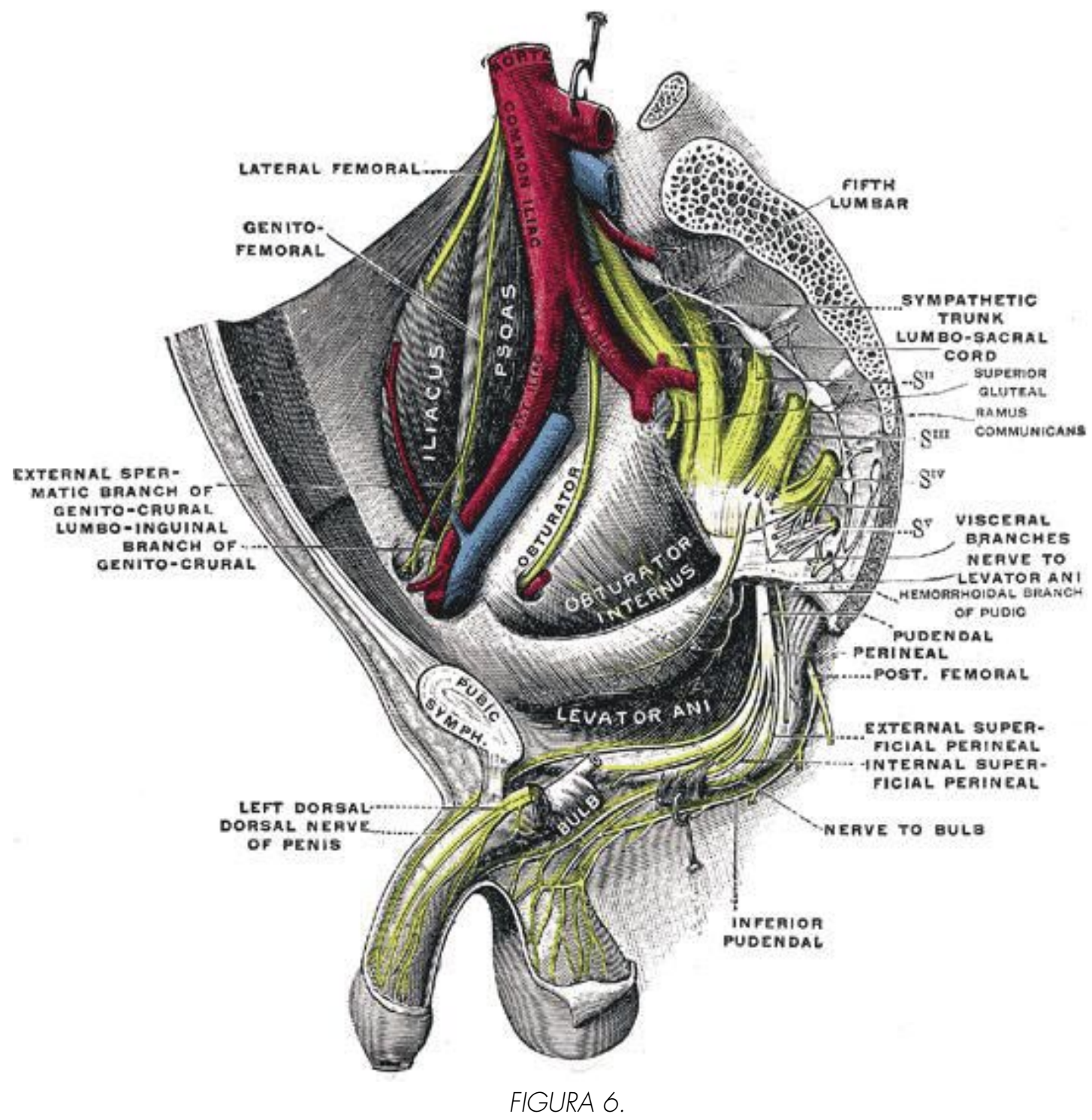




\section{Inervación parasimpática}

Las neuronas preganglionares involucradas en la actividad eferente parasimpática del pene están localizadas en la zona intermedia gris de la medula espinal, entre la $2^{\circ}$ y $4^{\circ}$ vertebra sacra. La estimulación de estos segmentos produce erección, siendo mayor a la altura de S2. Estas fibras preganglionares constituyen el plexo pélvico. Los nervios pélvicos también llevan información de la cadena simpática ganglionar sacra $(1,8)$.

Las fibras procedentes de los nervios hipogástricos se unen a los nervios pélvicos formando el plexo pélvico. Este plexo se constituye a modo de un plexo hipogástrico inferior situado en la fascia pélvica, a ambos lados del tracto genitourinario bajo y del recto (9).

Los nervios cavernosos son eferencias del plexo pélvico. Este plexo es una lámina cuadrilátera situada sagitalmente y lateral al recto, envuelta en un fascia vascular que depende de los vasos hipogástricos. Recibe aferencias del sistema nervioso autónomo simpático a través del nervio hipogástrico, de la cadena simpática sacra y de fibras nerviosas que provienen del plexo mesentérico inferior; y del sistema nervioso parasimpático a través de nervios esplácnicos pélvicos (10).

Las eferencias del plexo pélvico proveen inervación para el recto, vejiga, próstata, vesículas seminales, uretra y cuerpos cavernosos. La arteria vesical inferior perfora al plexo aproximadamente en su tercio inferior en dirección a la unión vesicoprostática, para luego irrigar el cuello vesical y la próstata. Casi la totalidad de las fibras nerviosas que quedan por debajo del punto de perforación del plexo por la mencionada arteria, contribuyen a formar el fascículo neurovascular prostático, que van a penetrar hacia el cuerpo esponjoso y a los cuerpos cavernosos para desencadenar los fenómenos neurovasculares durante la erección y la detumescencia (11).

Los nervios cavernosos se sitúan entre las dos hojas de la fascia endopélvica que podríamos denominar fascia periprostática (más interna) y fascia del elevador (más externa) (11).

Así mismo, los nervios cavernosos son eferencias del plexo pélvico y se ubican dentro de un fascículo neurovascular con una distribución variable alrededor de la próstata según cada caso, siendo un fascículo único o apareciendo dispersos por la superficie anterolateral de la próstata (1 1-13).

\section{BIBLIOGRAFÍA Y LECTURAS

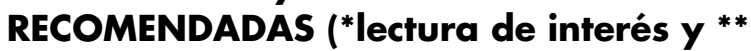 lectura fundamental)}

**1. Platas Sancho, Arturo. Anatomía Funcional del pene. En: Erección, eyaculación y sus trastornos.
Saenz de Tejada I, Allona A. Fomento Salud, SL. Madrid, España.1997.pp 1-12.

*2. Wein AJ, Kavousi LR, Novick AC, Partin AW, Peters CA. Campbell-Walsh Urology. 9th edition. Saunders, Elsevier 2007;61.

3. Prost H, Sharlip ID. Anatomy and Physiology of Erection. In:Standard Practice in Sexual Medicine. Prost H, Buvat J. Blackwell Publishing. 2006.

4. Breza J, Aboseif SR, Orvis BR, Lue TF, Tanagho EA. Detalled anatomy of penile neurovascular structures: surgical signifficance. J Urol 1989;141:437-443

5. Aboseif SR, Breza J, Lue TF, Tanagho EA: Penile venous drainage in erectile dysfunction: Anatomical, radiological and functional considerations. Br J Urol 1989;64:183-190.

*6. Bisschop E, Bautrant E. EMG: which one and why? considerations regarding pudendal nerve terminal motor latencies (pntml). 31th Meeting Annual of the International Urogynecological Association (IUGA 2006). Encontrado en internet: www.perineology.com/files/de-bisschopathens2006.pdf.

7. Spinosa JP, Bisschop E, Laurencon J, Kuhn G, Dubuisson JB, Riederer BM .Sacral staged reflexes to localize the pudendal compression: an anatomical validation of the concept. Rev Med Suisse. 2006 oct 25;2(84):2416-8, 2420-1.

8. Lepor H, Gregerman M, Ranice C, Mostofi FK, Walsh PC. Precise localization of the autonomic nerves from the pelvic plexus to the corpora cavernosa: a detail anatomical study of the adults male pelvis. J Urol 1984;133:207-212.

9. Tewari A, Peabody JO, Fischer M, Sarle R, Vallancien G, Delmas V, Hassan M, Bansal A, Hemal AK, Guillonneau B, Menon M. An operative and anatomic study to help in nerve sparing during laparoscopic and robotic radical prostatectomy. Eur Urol 2003;43(5):444-454

10. Eichelberg C, Erbersdobler A, Michl U, Schlomm T, Salomon G, Graefen M, Huland H. Nerve distribution along the prostatic capsule. Eur Urol 2007;51(1):105-111.

**11. Monzó Gardiner Juan I., Herranz Amo Felipe, Cabello Benavente Ramiro, Bun Maximiliano, Gómez Muñoz Jesús, González Enguita Carmen. Controversias en la anatomía quirúrgica prostática: Revisión de la literatura. Actas Urol Esp 2009; 33(3): 228-234

12. Walsh PC, Schlegel PN. Radical pelvic surgery with preservation of sexual function. Annals of Surgery 1988;208(4):391-400.

13. Monzó JI, Cabello, R, Moncada I, Herranz F, Hernandez C, Mazza O. Cadaveric dissection of cavernous nerves: its usefulness for retropubic radical prostatectomy. Ital J Sex Reprod Med 2007;14:63-108. 